PROCEEDINGS OF THE

AMERICAN MATHEMATICAL SOCIETY

Volume 129, Number 3, Pages 845-853

S 0002-9939(00)05988-8

Article electronically published on November 8, 2000

\title{
SOME RESULTS FOR ASYMPTOTICALLY PSEUDO-CONTRACTIVE MAPPINGS AND ASYMPTOTICALLY NONEXPANSIVE MAPPINGS
}

\author{
S. S. CHANG
}

(Communicated by Jonathan M. Borwein)

\begin{abstract}
Some convergence theorems of modified Ishikawa and Mann iterative sequences with errors for asymptotically pseudo-contractive and asymptotically nonexpansive mappings in Banach space are obtained. The results presented in this paper improve and extend the corresponding results in Goebel and Kirk (1972), Kirk (1965), Liu (1996), Schu (1991) and Chang et al. (to appear).
\end{abstract}

\section{INTRODUCTION AND PRELIMINARIES}

Throughout this paper, we assume that $E$ is a real Banach space, $E^{*}$ is the topological dual space of $E,\langle\cdot, \cdot\rangle$ is the dual pair between $E$ and $E^{*}, D(T), F(T)$ denote the domain of $T$ and the set of all fixed points of $T$ respectively and $J: E \rightarrow$ $2^{E^{*}}$ is the normalized duality mapping defined by

$$
J(x)=\left\{f \in E^{*}:\langle x, f\rangle=\|x\| \cdot\|f\|,\|f\|=\|x\|\right\}, \quad x \in E .
$$

Definition 1.1. Let $T: D(T) \subset E \rightarrow E$ be a mapping.

(1) $T$ is said to be asymptotically nonexpansive if there exists a sequence $\left\{k_{n}\right\}$ in $(0, \infty)$ with $\lim _{n \rightarrow \infty} k_{n}=1$ such that

$$
\left\|T^{n} x-T^{n} y\right\| \leq k_{n}\|x-y\|
$$

for all $x, y \in D(T)$ and $n=1,2, \ldots$.

(2) $T$ is said to be asymptotically pseudo-contractive if there exists a sequence $\left\{k_{n}\right\}$ in $(0, \infty)$ with $\lim _{n \rightarrow \infty} k_{n}=1$ and for any $x, y \in D(T)$ there exists $j(x-y) \in J(x-y)$ such that

$$
\left\langle T^{n} x-T^{n} y, j(x-y)\right\rangle \leq k_{n}\|x-y\|^{2}
$$

for all $n=1,2, \ldots$.

Received by the editors May 25, 1999.

1991 Mathematics Subject Classification. Primary 47H05, 47H10, 47H15.

Key words and phrases. Asymptotically nonexpansive mapping, asymptotically pseudocontractive mapping, modified Isikawa iterative sequence with errors, modified Mann iterative sequence with error, fixed point.

This paper was completed while the author visited Korea and was supported by the Korean Science and Engineering Foundation and National Natural Science Foundation of China. 
The following proposition follows from Definition 1.1 immediately.

Proposition 1.1. (1) If $T: D(T) \subset E \rightarrow E$ is nonexpansive, then $T$ is an asymptotically nonexpansive mapping with a constant sequence $\{1\}$.

(2) If $T: D(T) \subset E \rightarrow E$ is asymptotically nonexpansive, then $T$ is an asymptotically pseudo-contractive mapping. But the converse is not true in general.

This can be seen from the following example.

Example 1.1 ([8]). Let $E=\mathbb{R}$ and $D=[0,1]$, and let the mapping $T: D \rightarrow D$ be defined by

$$
T x=\left(1-x^{\frac{2}{3}}\right)^{\frac{3}{2}}
$$

for all $x \in D$. It can be proved that $T$ is not Lipschitzian [8], and so it is not asymptotically nonexpansive. Since $T$ is monotonically decreasing and $T \circ T=I$, we have

$$
\left(T^{n} x-T^{n} y\right)(x-y)= \begin{cases}|x-y|^{2}, & \text { if } n \text { is even; } \\ (T x-T y)(x-y) \leq 0 \leq|x-y|^{2}, & \text { if } n \text { is odd }\end{cases}
$$

This implies that $T$ is an asymptotically pseudo-contractive mapping with a constant sequence $\{1\}$.

Definition 1.2. (1) Let $T: D(T) \subset E \rightarrow E$ be a mapping, let $D(T)$ be a nonempty convex subset of $E$, let $x_{0} \in D(T)$ be a given point, and let $\left\{\alpha_{n}\right\},\left\{\beta_{n}\right\},\left\{\gamma_{n}\right\}$ and $\left\{\delta_{n}\right\}$ be four sequences in $[0,1]$. Then the sequence $\left\{x_{n}\right\}$ defined by

$$
\left\{\begin{array}{l}
x_{n+1}=\left(1-\alpha_{n}-\gamma_{n}\right) x_{n}+\alpha_{n} T^{n} y_{n}+\gamma_{n} u_{n} \\
y_{n}=\left(1-\beta_{n}-\delta_{n}\right) x_{n}+\beta_{n} T^{n} x_{n}+\delta_{n} v_{n}
\end{array} \quad \text { for all } n \geq 0\right.
$$

is called the modified Ishikawa iterative sequence with errors of $T$, where $\left\{u_{n}\right\}$ and $\left\{v_{n}\right\}$ are two bounded sequences in $D(T)$.

(2) In (1.1) if $\beta_{n}=0$ and $\delta_{n}=0, n=0,1,2, \ldots$, then $y_{n}=x_{n}$. The sequence $\left\{x_{n}\right\}$ defined by

$$
x_{n+1}=\left(1-\alpha_{n}-\gamma_{n}\right) x_{n}+\alpha_{n} T^{n} x_{n}+\gamma_{n} u_{n}, \quad n \geq 0,
$$

is called the modified Mann iterative sequence with errors of $T$.

The concept of asymptotically nonexpansive mapping was introduced by Goebel and Kirk [5] in 1972, which was closely related to the theory of fixed points of mappings in Banach spaces. An early fundamental result due to Goebel and Kirk [5] showed that if $E$ is a uniformly convex Banach space, $D$ is a nonempty bounded closed convex subset of $E$ and $T: D \rightarrow D$ is an asymptotically nonexpansive mapping, then $T$ has a fixed point in $D$. This result is a generalization of the corresponding results in Browder [1] and Kirk [6].

The concept of asymptotically pseudo-contractive mapping was introduced by Schu 9] in 1991.

The iterative approximation problems for nonexpansive mapping, asymptotically nonexpansive mapping and asymptotically pseudo-contractive mapping were studied extensively by Browder [1], Goebel and Kirk [5], Kirk [6], Liu [7, Rhoades [8], Schu [9], Xu [10, 11] and Xu and Roach [12] in the setting of Hilbert space or uniformly convex Banach spaces.

The purpose of this paper is to study the iterative approximation problems of fixed points for asymptotically pseudo-contractive mappings and asymptotically 
nonexpansive mappings in uniformly smooth Banach spaces by using a new iterative technique. The results presented in this paper extend and improve the main results in [3- 7], 9], and the methods of proof given in this paper are also quite different.

The following lemmas play an important role in this paper.

Lemma $1.1([2])$. Let $E$ be a real Banach space and $J: E \rightarrow 2^{E^{*}}$ a normalized duality mapping. Then for all $x, y \in E$

$$
\|x+y\|^{2} \leq\|x\|^{2}+2\langle y, j(x+y)\rangle
$$

for all $j(x+y) \in J(x+y)$.

Lemma 1.2 (12, p. 350]). Let $E$ be a uniformly convex real Banach space, $D$ a nonempty closed convex subset of $E$ and $T: D \rightarrow D$ a nonexpansive mapping. If $F(T) \neq \varnothing$, then for any $x \in D, q \in F(T)$ and any $j(x-q) \in J(x-q)$ the equality

$$
\langle T x-q, j(x-q)\rangle-\|x-q\|^{2}=0
$$

holds if and only if $x=q$.

\section{MAIN RESUlts}

Theorem 2.1. Let $E$ be a real uniformly smooth Banach space, let $D$ be a nonempty bounded closed convex subset of $E$, let $T: D \rightarrow D$ be an asymptotically pseudo-contractive mapping with a sequence $\left\{k_{n}\right\} \subset(0, \infty), \lim _{n \rightarrow \infty} k_{n}=1$, and let $F(T) \neq \varnothing$. Let $\left\{\alpha_{n}\right\},\left\{\beta_{n}\right\},\left\{\gamma_{n}\right\}$ and $\left\{\delta_{n}\right\}$ be four sequences in $[0,1]$ satisfying the following conditions:

(i) $\alpha_{n}+\gamma_{n} \leq 1, \beta_{n}+\delta_{n} \leq 1$;

(ii) $\alpha_{n} \rightarrow 0, \beta_{n} \rightarrow 0, \delta_{n} \rightarrow 0(n \rightarrow \infty)$;

(iii) $\sum_{n=0}^{\infty} \alpha_{n}=\infty, \sum_{n=0}^{\infty} \gamma_{n}<\infty$.

Let $x_{0} \in D$ be any given point and let $\left\{x_{n}\right\},\left\{y_{n}\right\}$ be the modified Ishikawa iterative sequence with errors defined by (1.1).

(1) If $\left\{x_{n}\right\}$ converges strongly to a fixed point $q$ of $T$ in $D$, then there exists a nondecreasing function $\phi:[0, \infty) \rightarrow[0, \infty), \phi(0)=0$ such that

$$
\left\langle T^{n} y_{n}-q, J\left(y_{n}-q\right)\right\rangle \leq k_{n}\left\|y_{n}-q\right\|^{2}-\phi\left(\left\|y_{n}-q\right\|\right),
$$

for all $n \geq 0$.

(2) Conversely, if there exists a strictly increasing function $\phi:[0, \infty) \rightarrow[0, \infty)$, $\phi(0)=0$ satisfying condition (2.1), then $x_{n} \rightarrow q \in F(T)$.

Proof. Since $E$ is uniformly smooth, the normalized duality mapping $J: E \rightarrow E^{*}$ is single-valued and uniformly continuous on any bounded subset of $E$.

"Necessity". Let $x_{n} \rightarrow q \in F(T)$. Since $D$ is a bounded set in $E$ and $\left\{T^{n} x_{n}\right\}$, $\left\{T^{n} y_{n}\right\},\left\{u_{n}\right\}$ and $\left\{v_{n}\right\}$ are sequences in $D$, they are therefore bounded. Besides since $\beta_{n} \rightarrow 0, \delta_{n} \rightarrow 0$, we have

$$
y_{n}=\left(1-\beta_{n}-\delta_{n}\right) x_{n}+\beta_{n} T^{n} x_{n}+\delta_{n} v_{n} \rightarrow q \quad(n \rightarrow \infty) .
$$

Define

$$
K=\sup _{n \geq 0}\left\{\left\|y_{n}-q\right\|\right\}<\infty .
$$

If $K=0$, then $y_{n}=q$ for all $n \geq 0$. Hence (2.1) is true for all $n \geq 0$; 
If $K>0$, define

$$
\begin{aligned}
& G_{t}=\left\{n \in \mathbb{N}:\left\|y_{n}-q\right\| \geq t\right\}, \quad t \in(0, K), \\
& G_{K}=\left\{n \in \mathbb{N}:\left\|y_{n}-q\right\|=K\right\},
\end{aligned}
$$

where $\mathbb{N}$ is the set of all nonnegative integers.

Since $y_{n} \rightarrow q$, for any $t \in(0, K]$ there exists $n_{0} \in \mathbb{N}$ such that for any $n \geq n_{0}$

$$
\left\|y_{n}-q\right\|<t
$$

This implies that for each $t \in(0, K)$

(a) $G_{t}$ is a nonempty finite subset of $\mathbb{N}$;

(b) $G_{t_{1}} \subset G_{t_{2}}$, if $t_{1} \geq t_{2}, t_{1}, t_{2} \in(0, K)$;

(c) $G_{K}=\bigcap_{t \in(0, K)} G_{t}$.

Since $T: D \rightarrow D$ is asymptotically pseudo-contractive, for given $q \in F(T)$ and for any $y_{n}$, we have

$$
\left\langle T^{n} y_{n}-q, J\left(y_{n}-q\right)\right\rangle \leq k_{n}\left\|y_{n}-q\right\|^{2}, \quad n \geq 0 .
$$

By virtue of (2.2), we define a function

$$
g(t)=\min _{n \in G_{t}}\left\{k_{n}\left\|y_{n}-q\right\|^{2}-\left\langle T^{n} y_{n}-q, J\left(y_{n}-q\right)\right\rangle\right\}, \quad t \in(0, K) .
$$

From (2.2) and property (b), we know that

(a) $g(t) \geq 0$ for all $t \in(0, K)$;

(b) $g(t)$ is nondecreasing in $t \in(0, K)$.

Next we define a function

$$
\phi(t)= \begin{cases}0, & \text { if } t=0, \\ g(t), & \text { if } t \in(0, K), \\ \lim _{s \rightarrow K-} g(s), & \text { if } t \in[K, \infty) .\end{cases}
$$

Hence $\phi:[0, \infty) \rightarrow[0, \infty)$ is nondecreasing and $\phi(0)=0$. For any $n \geq 0$, let $t_{n}=\left\|y_{n}-q\right\|$.

(1) If $t_{n}=0$, then $y_{n}=q$, hence $\phi\left(\left\|y_{n}-q\right\|\right)=0$, and so

$$
\left\langle T^{n} y_{n}-q, J\left(y_{n}-q\right)\right\rangle=k_{n}\left\|y_{n}-q\right\|^{2}-\phi\left(\left\|y_{n}-q\right\|\right) .
$$

(2) If $t_{n} \in(0, K)$, then $n \in G_{t_{n}}$, and so

$$
\begin{aligned}
\phi\left(\left\|y_{n}-q\right\|\right) & =g\left(t_{n}\right)=\min _{m \in G_{t_{n}}}\left\{k_{m}\left\|y_{m}-q\right\|^{2}-\left\langle T^{m} y_{m}-q, J\left(y_{m}-q\right)\right\rangle\right\} \\
& \leq k_{n}\left\|y_{n}-q\right\|^{2}-\left\langle T^{n} y_{n}-q, J\left(y_{n}-q\right)\right\rangle .
\end{aligned}
$$

(3) If $t_{n}=K$, then $n \in G_{K}=\bigcap_{s \in(0, K)} G_{s}$, and so

$$
\begin{aligned}
\phi\left(\left\|y_{n}-q\right\|\right) & =\phi\left(t_{n}\right)=\lim _{s \rightarrow K-} g(s) \\
& =\lim _{s \rightarrow K-} \min _{m \in G_{s}}\left\{k_{m}\left\|y_{m}-q\right\|^{2}-\left\langle T^{m} y_{m}-q, J\left(y_{m}-q\right)\right\rangle\right\} \\
& \leq k_{n}\left\|y_{n}-q\right\|^{2}-\left\langle T^{n} y_{n}-q, J\left(y_{n}-q\right)\right\rangle .
\end{aligned}
$$

The necessity is proved. 
"Sufficiency." From Lemma 1.1 and condition (2.1) we have

$$
\begin{aligned}
\left\|x_{n+1}-q\right\|^{2}= & \left\|\left(1-\alpha_{n}-\gamma_{n}\right)\left(x_{n}-q\right)+\alpha_{n}\left(T^{n} y_{n}-q\right)+\gamma_{n}\left(u_{n}-q\right)\right\|^{2} \\
\leq & \left(1-\alpha_{n}-\gamma_{n}\right)^{2}\left\|x_{n}-q\right\|^{2}+2 \alpha_{n}\left\langle T^{n} y_{n}-q, J\left(x_{n+1}-q\right)\right\rangle \\
& +2 \gamma_{n}\left\langle u_{n}-q, J\left(x_{n+1}-q\right)\right\rangle \\
\leq & \left(1-\alpha_{n}\right)^{2}\left\|x_{n}-q\right\|^{2}+2 \alpha_{n}\left\langle T^{n} y_{n}-q, J\left(x_{n+1}-q\right)-J\left(y_{n}-q\right)\right\rangle \\
& +2 \alpha_{n}\left\langle T^{n} y_{n}-q, J\left(y_{n}-q\right)\right\rangle+2 \gamma_{n}\left\langle u_{n}-q, J\left(x_{n+1}-q\right)\right\rangle .
\end{aligned}
$$

Now we consider the second term on the right side of 2.3. Since $\left\{T^{n} y_{n}-y_{n}\right\}$, $\left\{x_{n}-T^{n} x_{n}\right\},\left\{x_{n}-v_{n}\right\}$ and $\left\{u_{n}-y_{n}\right\}$ all are bounded and

$$
\begin{aligned}
x_{n+1}- & q-\left(y_{n}-q\right)=x_{n+1}-y_{n} \\
= & \left(1-\alpha_{n}-\gamma_{n}\right)\left(x_{n}-y_{n}\right)+\alpha_{n}\left(T^{n} y_{n}-y_{n}\right)+\gamma_{n}\left(u_{n}-y_{n}\right) \\
= & \left(1-\alpha_{n}-\gamma_{n}\right)\left\{\beta_{n}\left(x_{n}-T^{n} x_{n}\right)+\delta_{n}\left(x_{n}-v_{n}\right)\right\} \\
& +\alpha_{n}\left(T^{n} y_{n}-y_{n}\right)+\gamma_{n}\left(u_{n}-y_{n}\right),
\end{aligned}
$$

it follows that $x_{n+1}-q-\left(y_{n}-q\right) \rightarrow \theta$ (as $\left.n \rightarrow \infty\right)$. By the uniform continuity of $J$ and the boundedness of $\left\{T^{n} y_{n}-q\right\}$, we know that

$$
p_{n}:=\left\langle T^{n} y_{n}-q, J\left(x_{n+1}-q\right)-J\left(y_{n}-q\right)\right\rangle \rightarrow 0 \quad(\text { as } n \rightarrow \infty) .
$$

Substituting (2.4) and (2.1) into (2.3), we have

$$
\begin{aligned}
\left\|x_{n+1}-q\right\|^{2} \leq(1 & \left.-\alpha_{n}\right)^{2}\left\|x_{n}-q\right\|^{2}+2 \alpha_{n} p_{n} \\
& +2 \alpha_{n}\left\{k_{n}\left\|y_{n}-q\right\|^{2}-\phi\left(\left\|y_{n}-q\right\|\right)\right\}+2 \gamma_{n} C,
\end{aligned}
$$

where $C=\sup _{n \geq 0}\left\{\left\|u_{n}-q\right\| \cdot\left\|x_{n+1}-q\right\|\right\}<\infty$.

Next we make an estimation for $\left\|y_{n}-q\right\|^{2}$ :

$$
\begin{aligned}
\left\|y_{n}-q\right\|^{2}= & \left\|\left(1-\beta_{n}-\delta_{n}\right)\left(x_{n}-q\right)+\beta_{n}\left(T^{n} x_{n}-q\right)+\delta_{n}\left(v_{n}-q\right)\right\|^{2} \\
\leq & \left(1-\beta_{n}-\delta_{n}\right)^{2}\left\|x_{n}-q\right\|^{2}+2 \beta_{n}\left\langle T^{n} x_{n}-q, J\left(y_{n}-q\right)\right\rangle \\
& \quad+2 \delta_{n}\left\langle v_{n}-q, J\left(y_{n}-q\right)\right\rangle \\
\leq & \left\|x_{n}-q\right\|^{2}+2 \beta_{n} M_{1}+2 \delta_{n} M,
\end{aligned}
$$

where

$$
\begin{gathered}
M=\sup _{n \geq 0}\left\{\left\|v_{n}-q\right\| \cdot\left\|y_{n}-q\right\|\right\}, \\
M_{1}=\sup _{n \geq 0}\left\{\left\|T^{n} x_{n}-q\right\| \cdot\left\|y_{n}-q\right\|\right\}<\infty .
\end{gathered}
$$

Substituting (2.6) into (2.5) and using $M_{2}=\sup _{n \geq 0}\left\|x_{n}-q\right\|^{2}$ to simplify we have

$$
\begin{aligned}
\| x_{n+1} & -q \|^{2} \\
\leq & \left(\left(1-\alpha_{n}\right)^{2}+2 \alpha_{n} k_{n}\right)\left\|x_{n}-q\right\|^{2} \\
& +2 \alpha_{n}\left\{p_{n}+2 \beta_{n} k_{n} M_{1}+2 \delta_{n} k_{n} M\right\}-2 \alpha_{n} \phi\left(\left\|y_{n}-q\right\|\right)+2 \gamma_{n} C \\
\leq & \left\|x_{n}-q\right\|^{2}-\alpha_{n} \phi\left(\left\|y_{n}-q\right\|\right) \\
& -\alpha_{n}\left\{\phi\left(\left\|y_{n}-q\right\|\right)-\left[-2+\alpha_{n}+2 k_{n}\right] M_{2}-2\left(p_{n}+2 \beta_{n} k_{n} M_{1}+2 \delta_{n} k_{n} M\right)\right\} \\
& +2 \gamma_{n} C .
\end{aligned}
$$


Let

$$
\sigma=\inf _{n \geq 0}\left\{\left\|y_{n}-q\right\|\right\} .
$$

Next we prove that $\sigma=0$. Suppose the contrary; if $\sigma>0$, then $\left\|y_{n}-q\right\| \geq \sigma>0$ for all $n \geq 0$. Hence $\phi\left(\left\|y_{n}-q\right\|\right) \geq \phi(\sigma)>0$. From (2.7) we have

$$
\begin{array}{r}
\left\|x_{n+1}-q\right\|^{2} \leq\left\|x_{n}-q\right\|^{2}-\alpha_{n} \phi(\sigma)-\alpha_{n}\left\{\phi(\sigma)-\left[-2+\alpha_{n}+2 k_{n}\right] M_{2}\right. \\
\left.-2\left(p_{n}+2 \beta_{n} k_{n} M_{1}+2 \delta_{n} k_{n} M\right)\right\}+2 \gamma_{n} C .
\end{array}
$$

Since $\alpha_{n} \rightarrow 0, \beta_{n} \rightarrow 0, \delta_{n} \rightarrow 0, p_{n} \rightarrow 0, k_{n} \rightarrow 1$, there exists $n_{1}$ such that for all $n \geq n_{1}$,

$$
\phi(\sigma)-\left[-2+\alpha_{n}+2 k_{n}\right] M_{2}-2\left(p_{n}+2 \beta_{n} k_{n} M_{1}+2 \delta_{n} k_{n} M\right)>0 .
$$

Hence from (2.8) we have

$$
\left\|x_{n+1}-q\right\|^{2} \leq\left\|x_{n}-q\right\|^{2}-\alpha_{n} \phi(\sigma)+2 \gamma_{n} C \text { for all } n \geq n_{1},
$$

i.e.,

$$
\alpha_{n} \phi(\sigma) \leq\left\|x_{n}-q\right\|^{2}-\left\|x_{n+1}-q\right\|^{2}+2 \gamma_{n} C \quad \text { for all } n \geq n_{1} .
$$

Therefore for any $m \geq n_{1}$ we have

$$
\begin{aligned}
\sum_{n=n_{1}}^{m} \alpha_{n} \phi(\sigma) & \leq\left\|x_{n_{1}}-q\right\|^{2}-\left\|x_{m+1}-q\right\|^{2}+2 C \sum_{n=n_{1}}^{m} \gamma_{n} \\
& \leq\left\|x_{n_{1}}-q\right\|^{2}+2 C \sum_{n=n_{1}}^{m} \gamma_{n} .
\end{aligned}
$$

Let $m \rightarrow \infty$; by condition (iii) we have

$$
\infty=\sum_{n=n_{1}}^{\infty} \alpha_{n} \phi(\sigma) \leq\left\|x_{n_{1}}-q\right\|^{2}+2 C \sum_{n=n_{1}}^{\infty} \gamma_{n} .
$$

This is a contradiction. By this contradiction, $\sigma=0$. Therefore there exists a subsequence $\left\{y_{n_{j}}\right\} \subset\left\{y_{n}\right\}$ such that

$$
y_{n_{j}} \rightarrow q \quad\left(\text { as } n_{j} \rightarrow \infty\right),
$$

i.e.,

$$
y_{n_{j}}=\left(1-\beta_{n_{j}}-\delta_{n_{j}}\right) x_{n_{j}}+\beta_{n_{j}} T^{n_{j}} x_{n_{j}}+\delta_{n_{j}} v_{n_{j}} \rightarrow q \quad\left(\text { as } n_{j} \rightarrow \infty\right) .
$$

Since $\alpha_{n} \rightarrow 0, \beta_{n} \rightarrow 0, \gamma_{n} \rightarrow 0, \delta_{n} \rightarrow 0$ and $\left\{T^{n_{j}} x_{n_{j}}\right\},\left\{T^{n_{j}} y_{n_{j}}\right\},\left\{u_{n_{j}}\right\},\left\{v_{n_{j}}\right\}$ are all bounded, this implies that

$$
x_{n_{j}} \rightarrow q \quad\left(\text { as } n_{j} \rightarrow \infty\right) .
$$

From (2.9) we have

$$
x_{n_{j}+1}=\left(1-\alpha_{n_{j}}-\gamma_{n_{j}}\right) x_{n_{j}}+\alpha_{n_{j}} T^{n_{j}} y_{n_{j}}+\gamma_{n_{j}} u_{n_{j}} \rightarrow q \quad\left(\text { as } n_{j} \rightarrow \infty\right)
$$

and so

$$
\begin{aligned}
y_{n_{j}+1}= & \left(1-\beta_{n_{j}+1}-\delta_{n_{j}+1}\right) x_{n_{j}+1} \\
& +\beta_{n_{j}+1} T^{n_{j}+1} x_{n_{j}+1}+\delta_{n_{j}+1} v_{n_{j}+1} \rightarrow q \quad\left(\text { as } n_{j} \rightarrow \infty\right) .
\end{aligned}
$$

By induction we can prove that

$$
x_{n_{j}+i} \rightarrow q \quad \text { and } \quad y_{n_{j}+i} \rightarrow q \quad\left(\text { as } n_{j} \rightarrow \infty\right) \quad \text { for all } i \geq 0 .
$$

This implies that $x_{n} \rightarrow q$. This completes the proof of Theorem 2.1 
From Theorem 2.1 and Proposition 1.1 we can obtain the following theorem.

Theorem 2.2. Let $E$ be a real uniformly smooth Banach space, let $D$ be a nonempty bounded closed convex subset of $E$, let $T: D \rightarrow D$ be an asymptotically nonexpansive mapping with a sequence $\left\{k_{n}\right\} \subset(0, \infty), \lim _{n \rightarrow \infty} k_{n}=1$, and let $F(T) \neq \varnothing$. Let $\left\{\alpha_{n}\right\},\left\{\beta_{n}\right\},\left\{\gamma_{n}\right\}$ and $\left\{\delta_{n}\right\}$ be four real sequences in $[0,1]$ satisfying the conditions (i)-(iii) in Theorem 2.1. Let $x_{0}$ in $D$ be any given point and let $\left\{x_{n}\right\},\left\{y_{n}\right\}$ be the modified Ishikawa iterative sequence with errors defined by (1.1).

(1) If $\left\{x_{n}\right\}$ converges strongly to $q \in F(T)$, then there exists a nondecreasing function $\phi:[0, \infty) \rightarrow[0, \infty), \phi(0)=0$ such that

$$
\left\langle T^{n} y_{n}-q, J\left(y_{n}-q\right)\right\rangle \leq k_{n}\left\|y_{n}-q\right\|^{2}-\phi\left(\left\|y_{n}-q\right\|\right), \quad \text { for all } n \geq 0 .
$$

(2) Conversely, if there exists a strictly increasing function $\phi:[0, \infty) \rightarrow[0, \infty)$, $\phi(0)=0$ satisfying condition (2.10), then $x_{n} \rightarrow q \in F(T)$.

Theorem 2.3. Let $E$ be a uniformly convex and uniformly smooth real Banach space, let $D$ be a nonempty bounded closed convex subset of $E$, and let $T: D \rightarrow D$ be a nonexpansive mapping. Let $\left\{\alpha_{n}\right\},\left\{\beta_{n}\right\},\left\{\gamma_{n}\right\}$ and $\left\{\delta_{n}\right\}$ be four sequences in $[0,1]$ satisfying the conditions (i)-(iii) in Theorem 2.1. Let $\left\{x_{n}\right\},\left\{y_{n}\right\}$ be the modified Ishikawa iterative sequence with errors defined by (1.1). Then $\left\{x_{n}\right\}$ converges strongly to $q \in F(T)$ if and only if there exists a strictly increasing function $\phi:[0, \infty) \rightarrow[0, \infty), \phi(0)=0$ satisfying the following condition:

$$
\left\langle T^{n} y_{n}-q, J\left(y_{n}-q\right)\right\rangle \leq\left\|y_{n}-q\right\|^{2}-\phi\left(\left\|y_{n}-q\right\|\right) \quad \text { for all } n \geq 0 .
$$

Proof. Since $T: D \rightarrow D$ is a nonexpansive mapping, by Proposition 1.1 $T$ is an asymptotically nonexpansive mapping with a constant sequence $\{1\}$, and so it is also an asymptotically pseudo-contractive mapping with the same constant sequence $\{1\}$. By Goebel and Kirk [5, $F(T) \neq \varnothing$. Therefore the sufficiency of Theorem 2.3 follows from Theorem 2.1 immediately.

Next we prove the necessity of Theorem 2.3

Since $E$ is a uniformly smooth Banach space, the normalized duality mapping $J: E \rightarrow E^{*}$ is single-valued. Let $x_{n} \rightarrow q \in F(T)$. Since $\left\{T^{n} x_{n}\right\}$ and $\left\{v_{n}\right\}$ are bounded and $\beta_{n} \rightarrow 0, \delta_{n} \rightarrow 0$, we know that

$$
y_{n}=\left(1-\beta_{n}-\delta_{n}\right) x_{n}+\beta_{n} T^{n} x_{n}+\delta_{n} v_{n} \rightarrow q .
$$

By the same way as given in proving the necessity of Theorem 2.1, let us define

$$
\begin{aligned}
K & =\sup _{n \geq 0}\left\{\left\|y_{n}-q\right\|\right\}<\infty, \\
G_{t} & =\left\{n \in \mathbb{N}:\left\|y_{n}-q\right\| \geq t\right\}, \quad t \in(0, K), \\
G_{K} & =\left\{n \in \mathbb{N}:\left\|y_{n}-q\right\|=K\right\}, \\
g(t) & =\min _{n \in G_{t}}\left\{\left\|y_{n}-q\right\|^{2}-\left\langle T^{n} y_{n}-q, J\left(y_{n}-q\right)\right\rangle\right\}, \quad t \in(0, K) .
\end{aligned}
$$

In Theorem 2.1 we proved that $g(t)$ is nondecreasing and $g(t) \geq 0$ for all $t \in(0, K)$.

Next we prove that $g(t)>0$ for all $t \in(0, K)$.

Suppose the contrary; there exists $t_{0} \in(0, K)$ such that $g\left(t_{0}\right)=0$. Since $G_{t_{0}}$ is a finite set, there exists an $n_{0} \in G_{t_{0}}$ such that

$$
0=g\left(t_{0}\right)=\left\|y_{n_{0}}-q\right\|^{2}-\left\langle T^{n_{0}} y_{n_{0}}-q, J\left(y_{n_{0}}-q\right)\right\rangle .
$$

Since $T: D \rightarrow D$ is nonexpansive and $q \in F(T)$, hence $T^{n_{0}}: D \rightarrow D$ is also nonexpansive and $q \in F\left(T^{n_{0}}\right)$. By Lemma 1.2, it follows from (2.12) that $y_{n_{0}}=q$, 
i.e., $\left\|y_{n_{0}}-q\right\|=0$. However, since $n_{0} \in G_{t_{0}}$, by the definition of $G_{t_{0}}$, we have $\left\|y_{n_{0}}-q\right\| \geq t_{0}>0$. This is a contradiction. Therefore $g(t)>0$ for all $t \in(0, K)$.

Now we define a function

$$
\phi(t)= \begin{cases}0, & \text { if } t=0, \\ \frac{t}{1+t} g(t), & \text { if } t \in(0, K), \\ \frac{t}{1+t} \lim _{s \rightarrow K-} g(s), & \text { if } t \in[K, \infty) .\end{cases}
$$

Since $g$ is nondecreasing and $g(t)>0$ for all $t \in(0, K), \phi(t):[0, \infty) \rightarrow[0, \infty)$ is strictly increasing, $\phi(0)=0$. By the same way as given in the proof of Theorem 2.1 we can prove that $\phi$ satisfies condition (2.11).

This completes the proof of Theorem 2.3

Theorem 2.4. Let $E$ be a real uniformly smooth Banach space, let $D$ be a nonempty bounded closed convex subset of $E$, let $T: D \rightarrow D$ be an asymptotically pseudocontractive mapping with a sequence $\left\{k_{n}\right\} \subset(0, \infty), \lim _{n \rightarrow \infty} k_{n}=1$, and let $F(T) \neq \varnothing$. Let $\left\{\alpha_{n}\right\}$ and $\left\{\gamma_{n}\right\}$ be two real sequences in $[0,1]$ satisfying the following conditions:

(i) $\alpha_{n}+\gamma_{n} \leq 1$;

(ii) $\alpha_{n} \rightarrow 0(n \rightarrow \infty)$;

(iii) $\sum_{n=0}^{\infty} \alpha_{n}=\infty, \sum_{n=0}^{\infty} \gamma_{n}<\infty$.

Let $x_{0} \in D$ be any given point and let $\left\{x_{n}\right\}$ be the modified Mann iterative sequence with errors defined by (1.2).

(1) If $\left\{x_{n}\right\}$ converges strongly to a fixed point $q$ of $T$, then there exists a nondecreasing function $\phi:[0, \infty) \rightarrow[0, \infty), \phi(0)=0$ such that

$$
\left\langle T^{n} x_{n}-q, J\left(x_{n}-q\right)\right\rangle \leq k_{n}\left\|x_{n}-q\right\|^{2}-\phi\left(\left\|x_{n}-q\right\|\right), \quad \text { for all } n \geq 0 .
$$

(2) Conversely, if there exists a strictly increasing function $\phi:[0, \infty) \rightarrow[0, \infty)$, $\phi(0)=0$ satisfying condition (2.13), then $x_{n} \rightarrow q \in F(T)$.

Proof. Taking $\beta_{n}=\delta_{n}=0$ for all $n \geq 0$ in Theorem 2.1 we then have $y_{n}=x_{n}$ for all $n \geq 0$. Therefore the conclusion of Theorem 2.4 follows from Theorem 2.1 immediately.

Remark. Theorems 2.12.4 generalize the corresponding results in [3]-[7, 9].

\section{REFERENCES}

[1] F. E. Browder, Nonexpansive nonlinear operators in Banach spaces, Proc. Natl. Acad. Sci. USA, 54 (1965), 1041-1044. MR 32:4574

[2] Shih-sen Chang, Some problems and results in the study of nonlinear analysis, Nonlinear Anal. TMA, 30 (1997), 4197-4208. MR 99a:47089

[3] Shih-sen Chang, J. Y. Park and I. H. Jung, Some results on convergence of iterative methods for accretive and pseudo-contractive type mappings in Banach spaces, (submitted).

[4] Shih-sen Chang, J. Y. Park, Y. J. Cho and I. H. Jung, Iterative approximations of asymptotically pseudo-contractive mappings in Banach spaces, (submitted).

[5] K. Goebel and W. A. Kirk, A fixed point theorem for asymptotically nonexpansive mappings, Proc. Amer. Math. Soc., 35 (1) (1972), 171-174. MR 45:7552

[6] W. A. Kirk, A fixed point theorem for mappings which do not increase distance, Amer. Math. Monthly, 72 (1965), 1004-1006. MR 32:6436

[7] Q. H. Liu, Convergence theorems of the sequence of iterates for asymptotically demicontractive and hemi-contractive mappings, Nonlinear Anal. TMA, 26 (11) (1996), 18351842. MR 97d:47069 
[8] B. E. Rhoades, Comments on two fixed point iterative methods, J. Math. Anal. Appl., 56 (1976), 741-750. MR 55:3885

[9] J. Schu, Iterative construction of fixed points of asymptotically nonexpansive mappings, J. Math. Anal. Appl., 158 (1991), 407-413. MR 92d:47072

[10] H. K. Xu, Inequalities in Banach spaces with applications, Nonlinear Anal. TMA, 16 (12) (1991), 1127-1138. MR 92e:47126

[11] H. K. Xu, Existence and convergence for fixed points of mapping of asymptotically nonexpansive type, Nonlinear Anal. TMA, 16 (12) (1991), 1139-1146. MR 92h:47089

[12] Z. B. Xu and G. F. Roach, A necessary and sufficient condition for convergence of steepest descent approximation to accretive operator equations, J. Math. Anal. Appl., 167 (1992), 340-354. MR 93e:47086

Department of Mathematics, Sichuan University, Chengdu, Sichuan 610064, People's Republic of China

E-mail address: sszhang@mail.sc.cninfo.net 